\title{
Modification Processes for Highly Hydrogenated Diamond-Like Carbon Thin Films by Soft X-ray Irradiation
}

\author{
Kazuhiro Kanda, Ryo Imai, Masahito Niibe, \\ Hisashi Yoshioka, ${ }^{1}$ Keishi Komatsu, ${ }^{1}$ and Hidetoshi Saitoh ${ }^{1}$ \\ Laboratory of Advanced Science and Technology for Industry, University of Hyogo, \\ 3-1-2 Koto, Kamigori-cho, Ako-gun, Hyogo 678-1205, Japan \\ ${ }^{1}$ Graduate School of Engineering, Nagaoka University of Technology, \\ 16031-1 Kamitomioka, Nagaoka, Niigata 940-2188, Japan
}

(Received September 23, 2016; accepted December 26, 2016)

Keywords: diamond-like carbon, hydrogen desorption, soft X-ray, film modification, NEXAFS

We investigated a modification process for highly hydrogenated diamond-like carbon (H-DLC) film using exposure to synchrotron radiation (SR) in the soft X-ray region by measuring the dependence of various film properties and structural properties on the SR dose. Noticeable changes in the film volume and optical constants were observed, and the local structures of the H-DLC film changed due to SR exposure. These changes are ascribable to the desorption of hydrogen from the film, which is much faster than when the film is etched.

\section{Introduction}

Diamond-like carbon (DLC) film is amorphous carbon film that contains $s p^{3}$ hybridized carbon atoms corresponding to a diamond structure and $s p^{2}$ hybridized carbon atoms corresponding to a graphite structure. In addition, DLC film usually contains a certain amount of hydrogen. The superlubricity of DLC film, along with its low friction coefficient, high hardness, chemical inertness, and high wear and corrosion resistance, make it one of the most promising coatings for applications such as cutting tools, automobile parts, molds, computer hard disks, optical devices, food containers, and artificial blood vessels. ${ }^{(1-5)}$ Recently, DLC film has been considered as a lubricant for use in space due to its superlubricity. Oils and fats, which are used as lubricants on the ground, cannot be used in space because they evaporate and freeze in a vacuum. Hydrogenated DLC (H-DLC) film with a hydrogen content greater than 40 at.\% is expected to be a suitable lubricant in space because it provides ultra-low friction (friction coefficient less than 0.001), even under vacuum conditions. ${ }^{(6-8)}$ On the other hand, environmental conditions in space require tolerances very different from those on Earth, such as ultra-high vacuum, variations in temperature (from high temperature to low temperature), collisions with ionic particles and neutral atoms, and soft X-ray irradiation. DLC film generally has a very strong tolerance for soft X-ray irradiation. ${ }^{(9)}$ However, our group has recently reported that exposure of H-DLC film to soft X-rays leads to surface modifications; Irradiation of highly hydrogenated DLC film by soft X-rays decreases the film volume and hydrogen content ${ }^{(10)}$ and increases hardness. ${ }^{(11)}$ The film volume and hardness change dramatically over a synchrotron radiation (SR) dose range of 0-200 $\mathrm{mA} \cdot \mathrm{h}$ and change *Corresponding author: e-mail: kanda@lasti.u-hyogo.ac.jp http://dx.doi.org/10.18494/SAM.2017.1476 
gradually at SR doses of greater than $200 \mathrm{~mA} \cdot \mathrm{h}$. In addition, the hydrogen content in H-DLC film decreases from $\approx 50$ to $\approx 30 \%$ due to SR exposure at $200 \mathrm{~mA} \cdot \mathrm{h}$, but $\approx 20 \%$ of hydrogen remains in the film even after SR exposure at $3000 \mathrm{~mA} \cdot \mathrm{h}$. The modification processes caused by exposure of H-DLC films to soft X-rays are not yet fully understood.

To use H-DLC film safely in space, it is necessary to clarify the effect of soft X-ray irradiation on H-DLC film properties and the rate of deterioration of H-DLC films due to irradiation by soft X-rays. To understand the modification processes due to soft X-ray irradiation of H-DLC film, we measured the dependence of other film properties on the SR dose, e.g., density and optical constants. Optical constants, such as the refractive index $(n)$ and extinction coefficient $(k)$ of DLC film, have attracted attention as convenient indices of DLC structure. ${ }^{(12)}$ In addition, the dependence of the local structure of carbon on the SR dose was investigated to clarify the reason for the changes in film properties. Furthermore, the species desorbed from H-DLC film due to SR exposure were detected to understand the reactions caused by exposure to the soft-X rays.

\section{Material and Methods}

Hydrogenated DLC film was deposited on Si wafers using an amplitude-modulated radiofrequency plasma-enhanced chemical vapor deposition method (Nippon ITF Co.). ${ }^{(13)}$ This method enables the deposition of DLC film containing a large amount of hydrogen. The desired film thickness was $200 \mathrm{~nm}$. The hydrogen content of H-DLC film was estimated to be $\approx 50$ at. $\%$ using a combination of Rutherford backscattering spectrometry (RBS) and elastic recoil detection analysis (ERDA). ${ }^{(11)}$

The irradiation of the H-DLC film by SR was carried out at BL06 of the NewSUBARU synchrotron facility. ${ }^{(14)}$ The experimental apparatuses at BL06 are described in detail in previous reports. ${ }^{(15,16)}$ The SR extracted from a bending magnet, which is the light source of BL06, was introduced to the sample stage as a straight beam via a pair of mirrors. The SR at the BL06 sample stage had a continuous spectrum from infrared to soft X-rays below $1 \mathrm{keV}$. This energy range includes $300 \mathrm{eV}$, which is the ionization energy of a carbon $K$ shell. During this experiment, the electron energy of the NewSUBARU ring was $1.0 \mathrm{GeV}$ and the ring current was $300 \mathrm{~mA}$ in the top-up mode. Except for the detection of desorbed species using a quadrupole mass spectrometer, the SR was irradiated onto the film surface perpendicularly. The SR dose $[\mathrm{mA} \cdot \mathrm{h}]$ is derived from the product of the ring current (mA) and exposure time (h). During exposure of the H-DLC film to soft X-rays, the pressure in the irradiation chamber was on the order of $10^{-5} \mathrm{~Pa}$ and the temperature of the sample stage was confirmed to be room temperature using a thermocouple. After SR exposure, the modified H-DLC film was kept in a dry box.

\section{Results}

To discuss the modification processes due to soft X-ray irradiation of H-DLC film, we measured the dependence of the species desorbed from the H-DLC film surface on the dose of soft X-ray irradiation and the various film properties of the H-DLC film after irradiation. The film density and thickness were estimated from X-ray reflection (XRR) measurements. The local structure of the H-DLC film was discussed based on the $\mathrm{C} K$-edge near-edge X-ray absorption fine structure (NEXAFS) spectrum using SR. Optical coefficients $n$ and $k$ of the DLC film were obtained using a 
spectroscopic ellipsometer (SE). The surface composition was obtained from X-ray photoelectron spectroscopy (XPS). The species desorbed from the H-DLC film surface by irradiation with soft X-rays were measured using a quadruple mass spectrometer. In this study, the H-DLC film was exposed to SR in the soft X-ray in the dose region from 0 to $3000 \mathrm{~mA} \cdot \mathrm{h}$.

\subsection{Film thickness and density from X-ray reflection measurements}

We carried out XRR measurements to obtain the thickness and density of the H-DLC films. The XRR was measured with an XRR apparatus (Mac Science, M03XHF MXP3). Figure 1 shows the exposure-dose dependence of film thickness estimated from the XRR measurements. The film thickness before irradiation was determined under the film formation conditions, i.e., $\approx 200 \mathrm{~nm}$. The film thickness decreased suddenly from $\approx 200 \mathrm{~nm}$ to $\approx 145 \mathrm{~nm}$ due to SR exposure in the dose range of $0-200 \mathrm{~mA} \cdot \mathrm{h}$. At doses greater than $200 \mathrm{~mA} \cdot \mathrm{h}$, the film thickness gradually decreased and remained constant at $130 \mathrm{~nm}$.

Figure 2 shows the dependence of film density on dose based on XRR measurements. The density of the H-DLC film before irradiation was $1.25 \mathrm{~g} / \mathrm{cm}^{3}$. The densities of diamond and graphite are 3.515 and $2.267 \mathrm{~g} / \mathrm{cm}^{3}$, respectively; that of DLC film is in the range of 1.6-3.1 $\mathrm{g} / \mathrm{cm}^{3},{ }^{(5)}$ and that of the H-DLC film used in this study was $1.25 \mathrm{~g} / \mathrm{cm}^{3}$. The density of the H-DLC film increased dramatically to $1.40 \mathrm{~g} / \mathrm{cm}^{3}$ after $200-\mathrm{mA} \cdot \mathrm{h}$ SR exposure. At doses of more than 200 $\mathrm{mA} \cdot \mathrm{h}$, the film density gradually increased and remained constant at $1.60 \mathrm{~g} / \mathrm{cm}^{3}$ at doses of more than $2000 \mathrm{~mA} \cdot \mathrm{h}$. From these results, we confirmed that H-DLC film thickness decreases and film density increases due to soft X-ray irradiation.

\subsection{Local structure of carbon atoms from NEXAFS measurement}

NEXAFS was used to evaluate the local structure of carbon materials. ${ }^{(17-19)}$ The technique determines the $s p^{2} / s p^{3}$ ratio of carbon atoms in DLC film with high accuracy, because the excitation from the 1 s orbital to $\pi$-character empty state can be separately observed. ${ }^{(20-22)}$ The $\mathrm{C}$ $K$-edge NEXAFS spectra of H-DLC films in our study were measured in the photon energy range of $270-330 \mathrm{eV}$.

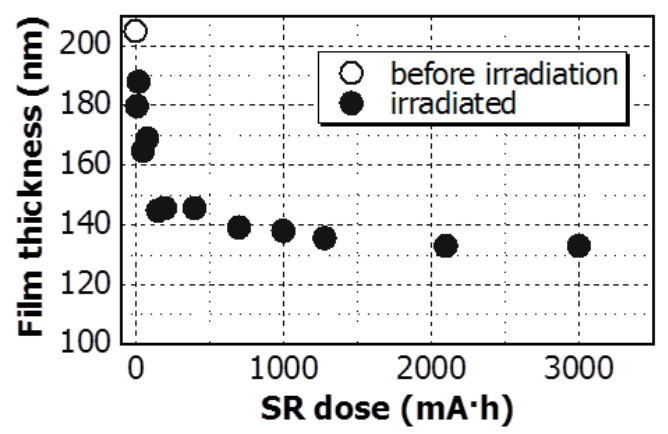

Fig. 1. Dependence of thickness of H-DLC film on SR dose. The open circle indicates thickness of H-DLC film before SR irradiation.

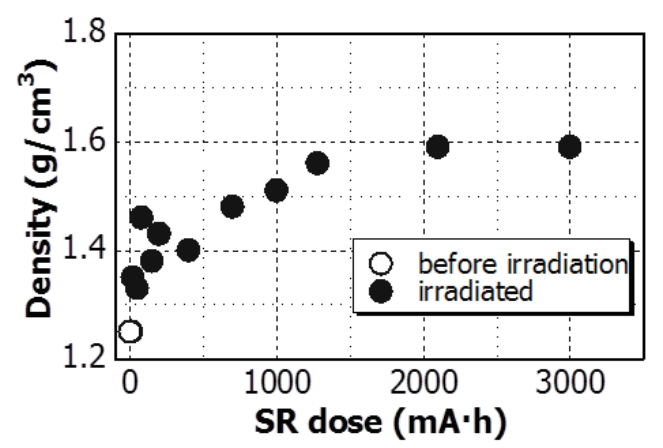

Fig. 2. Dependence of density of H-DLC film on SR dose. The open circle indicates density of H-DLC film before SR irradiation. 
The NEXAFS spectra were measured at BL09A of the NewSUBARU. The details of the measurement procedure for C K-edge NEXAFS are given in Refs. 23 and 24. The undulator light provided by an 11-m undulator was extracted using a varied line-spacing-plane grating of 1200 lines $/ \mathrm{mm}$ and was irradiated on a sample film at the magic angle $\left(54.7^{\circ}\right)$ with respect to the surface of the sample. The energy resolution was estimated to be less than $0.5 \mathrm{eV}$ full width at half maximum. The monochromator output energy was calibrated using the pre-edge position, which corresponds to the carbon $1 s \rightarrow \pi^{*}$ resonance transition observed at $285.38 \mathrm{eV}$ in the NEXAFS spectrum of graphite. ${ }^{(25)}$ Photocurrent from the sample was detected in the total electron yield mode. The intensity of the incident X-ray was measured by detecting the photocurrent from a gold mesh. The absorption signal was obtained from the ratio of the photocurrent from the sample to that from the gold mesh. The electron energy of the NewSUBARU ring was $1.5 \mathrm{GeV}$ during these measurements.

Figure 3 shows the C- $K$ edge NEXAFS spectra of the H-DLC film irradiated by SR, of the film before irradiation, and of a commercial DLC film as a typical DLC film. This commercial DLC film was deposited on $200 \mathrm{~nm}$-thick Si wafers using the ion plating method; thus we call it IP-DLC film. The hydrogen content of this IP-DLC film was estimated to be $\approx 20 \%$ from a combination of ERDA and RBS measurements. A sharp $\pi^{*}$ peak observed at $285.38 \mathrm{eV}$ is ascribed to the $\mathrm{C} 1 s \rightarrow \pi^{*}$ resonance transition originating from the carbon-carbon double bonding. A broad $\sigma^{*}$ peak observed at about $285-310 \mathrm{eV}$ is ascribed to the $\mathrm{C} 1 s \rightarrow \sigma^{*}$ resonance transition.

In the $C-K$ edge NEXAFS spectrum of the H-DLC film before irradiation, specific peaks were observed at around 290 and 300-305 eV, unlike the IP-DLC film, as shown in Fig. 3. The intensity of these specific peaks in the spectrum of the H-DLC film decreased with increasing SR dose, and these peaks disappeared in the spectrum of the H-DLC film after 200-mA.h SR exposure. As a result, the spectral features of H-DLC film after SR exposure of more than $200 \mathrm{~mA} \cdot \mathrm{h}$ resembled that of the IP-DLC film. In other words, the SR exposure of the H-DLC film led to a structural

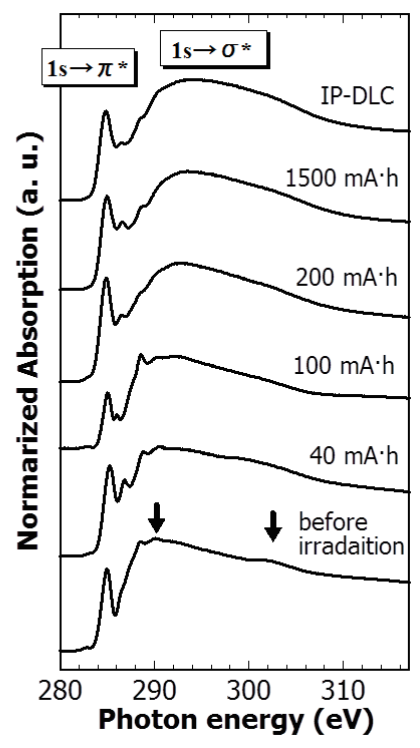

Fig. 3. Dependence of C $K$-edge NEXAFS spectra of H-DLC and commercial DLC films on SR dose. Arrows in the spectrum of H-DLC film before irradiation indicate specific peaks that decreased with increasing SR dose. 
change making it closer to typical DLC film. These changes in the C- $K$ edge NEXAFS spectra are believed to correspond to hydrogen desorption from the film. As described in Ref. 11, hydrogen content in H-DLC films decreases from $\approx 50$ to $\approx 30 \%$ due to 200 -mA.h SR exposure, and $\approx 20 \%$ of the hydrogen is not desorbed due to SR exposure. The specific peaks described here are ascribable to carbon atoms bonding to hydrogen atoms that were desorbed due to SR exposure of the H-DLC film. In other words, the unoccupied states of carbon atoms bonded to hydrogen atoms that were desorbed due to SR exposure were different from those of carbon atoms bonded to hydrogen atoms which were not desorbed due to SR exposure.

The absolute $s p^{2} /\left(s p^{2}+s p^{3}\right)$ ratio of carbon atoms in the DLC film can be determined with high accuracy, as described in Ref. 20. Figure 4 shows the SR dose-dependence of the $s p^{2} /\left(s p^{2}\right.$ $+s p^{3}$ ) ratio in the H-DLC film. These ratios were estimated quantitatively from the C- $K$ edge NEXAFS spectra of the H-DLC film shown in Fig. 3. The amount of $s p^{2}$ bonded carbon atoms can be extracted by normalizing the area of the resonance corresponding to the $1 s \rightarrow \pi^{*}$ transitions at $285.4 \mathrm{eV}$ with a large section of the spectrum. The absolute $s p^{2} /\left(s p^{2}+s p^{3}\right)$ ratio was determined by comparing it with that from the NEXAFS spectrum of graphite. The $s p^{2} /\left(s p^{2}+s p^{3}\right)$ ratio of the H-DLC film before irradiation was $\approx 0.33$, and it increased rapidly at an SR dose of less than $200 \mathrm{~mA} \cdot \mathrm{h}$. However, at an SR dose above $200 \mathrm{~mA} \cdot \mathrm{h}$, the $s p^{2} /\left(s p^{2}+s p^{3}\right)$ ratio was approximately constant at $\approx 0.50$.

\subsection{Optical constants from spectroscopic elipsometry}

Optical constants of DLC film are recognized as an important factor reflecting the film's local structure. ${ }^{(26,27)}$ Recently, many researchers have reported the optical properties of DLC films using spectroscopic ellipsometry (SE), which is a powerful optical technique that enables the measurement and interpretation of changes in the polarization state of polarized light undergoing oblique reflection from a sample surface. ${ }^{(28-34)}$ The technique is used to determine the films reflective index, $n$, and extinction coefficient, $k$, film thickness, and the surface roughness. Recently, the relation of optical constants to the local structure of DLC films attracted attention. It was reported that the refractive index increased with increasing $s p^{3}$ content and decreasing $\mathrm{H}$ content, and the extinction coefficient increased with $s p^{2}$ content. ${ }^{(35)}$ We investigated the SR dosedependence of the optical properties of H-DLC film using SE.

Ellipsometric measurements were conducted with a spectroscopic ellipsometer incorporating non-rotating phase modulation and liquid crystal modulation technologies (Horiba, UVISEL). The spectra of the DLC films were obtained from 0.6 to $4.8 \mathrm{eV}$ in $0.05-\mathrm{eV}$ increments. The angle of incidence was $70^{\circ}$. The optical constants and film thicknesses were determined by fitting the ellipomeric measurements to a model structure, which layered a $\mathrm{Si}$ wafer, natural oxidized silicon layer, a-C, and mixture of a-C and voids from the bottom up. The SR-dose dependencies of $n$ and $k$ as derived from the model are shown in Fig. 5. The $k$ of the H-DLC film before SR exposure was very small, $0.0005-0.001$, and increased greatly due to SR exposure. It increased drastically to $\approx 0.2$ with an SR dose of $1000 \mathrm{~mA} \cdot \mathrm{h}$ and gradually increased to $\approx 0.3$ with an SR dose of 3000 $\mathrm{mA} \cdot \mathrm{h}$. The $n$ of the H-DLC film also increased due to SR exposure. It increased from $\approx 1.5$ before SR exposure to $\approx 2.0$ with an SR dose of $800 \mathrm{~mA} \cdot \mathrm{h}$ and remained constant beyond an SR dose of $800 \mathrm{~mA} \cdot \mathrm{h}$.

Recently, the combination of $n$ and $k$ (n-k plot), which is determined from SE, has received much attention regarding the structural index of DLC films. ${ }^{(12)}$ The values of $n$ and $k$ measured 


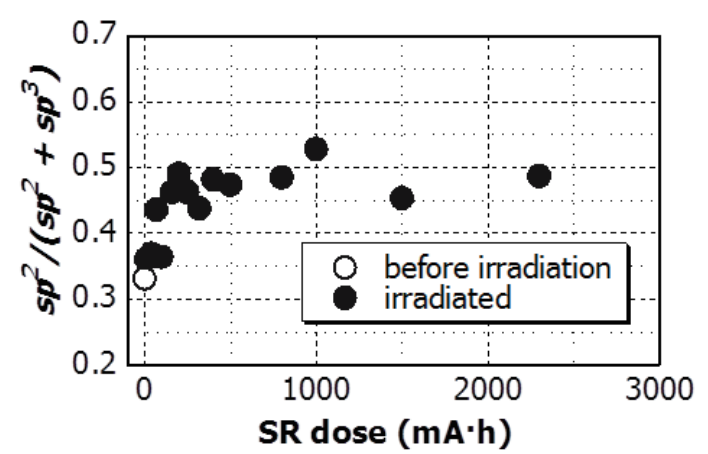

Fig. 4. Dependence of the $s p^{2} /\left(s p^{2}+s p^{3}\right)$ ratio in H-DLC film on SR dose. The open circle indicates the $s p^{2} /\left(s p^{2}+s p^{3}\right)$ ratio of H-DLC film before SR irradiation.

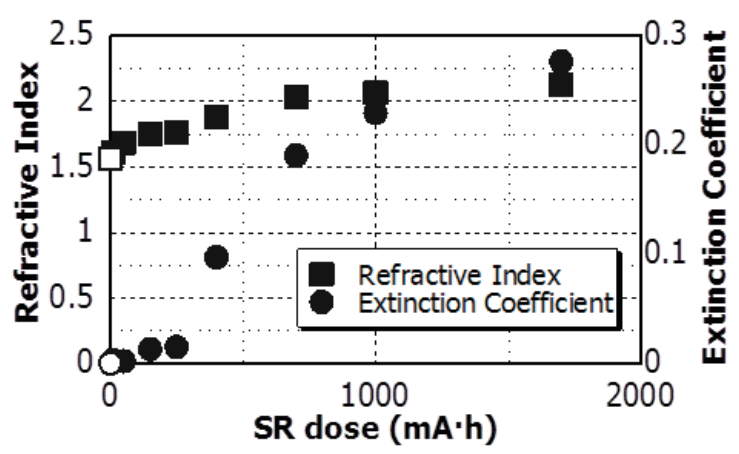

Fig. 5. Dependence of refractive index (circles) and extinction coefficient (squares) of H-DLC film on SR dose. The open circle and square indicate the refractive index and extinction coefficient of H-DLC film before SR irradiation, respectively.

at a wavelength of $550 \mathrm{~nm}$ are plotted in Fig. 6. The plot of the DLC film before SR exposure is located in the lower left-hand corner, which is characteristic of a polymer-like carbon film. This assignment was supported by the fact that the hydrogen content in this film was higher than $50 \%$. The $n$ and $k$ values increased due to SR exposure. The plot with $n=\approx 2.0$ and $k=\approx 0.3$ is characteristic of a-C:H film. Such a large change in $n$ and $k$ values of DLC film was observed in this study for the first time.

\subsection{Surface elementary composition by XPS}

Elementary composition of the H-DLC film surface was estimated from XPS measurements, which were carried out using a conventional photoelectron spectroscopy apparatus (Shimadzu, ESCA-1000) mounted with a hemispherical electron energy analyzer, CL150 (VSW Ltd.). The $\mathrm{Mg} \mathrm{K \alpha}$ line $(1253.6 \mathrm{eV})$ used as the X-ray source was incident at $45^{\circ}$ with respect to the surface normal. An electron emitted at $90^{\circ}$ with respect to the incident X-ray was detected. In addition to carbon, oxygen, which coupled with the H-DLC film surface as a result of surface oxidization, was observed in the XPS spectra. Figure 7 shows the SR dose dependence of the elementary composition of the H-DLC film estimated from the XPS spectra. The ratio of oxygen and carbon in the elementary composition of the H-DLC film hardly changed with increasing SR dose.

\subsection{Desorbed species from H-DLC film surface by soft X-ray irradiation}

The dose dependencies of species desorbed from the H-DLC film surface by soft $\mathrm{X}$-ray irradiation was measured using a quadruple mass spectrometer (QMS) (Anelva Co., Q-M400QA-M) installed in the irradiation chamber of BL06. The detectable mass range was from 1 to 400 , and mass resolution was $M / \Delta M \geq 2 \mathrm{M}$. The QMS was positioned perpendicular to the advancing direction of the soft X-ray beam. During this measurement, an incident soft X-ray beam was introduced to a sample at $45^{\circ}$ with respect to the surface normal. The species desorbed from the sample were detected with the QMS positioned at $45^{\circ}$ with respect to the surface normal of the sample in the opposite direction of the incident beam. The signals detected by the QMS were 


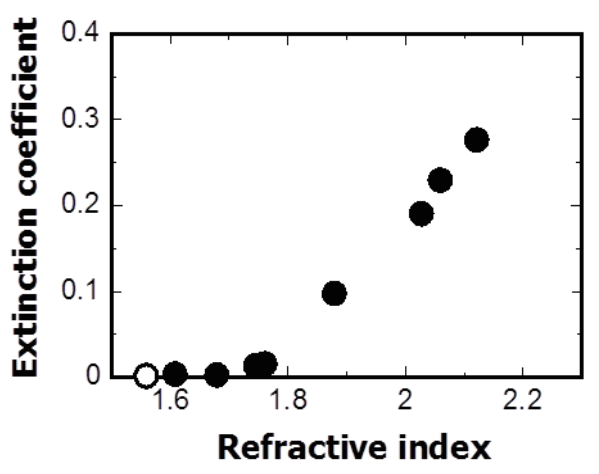

Fig. 6. The $n-k$ plot at $550 \mathrm{~nm}$ wavelength of an $\mathrm{H}$-DLC film. The open circle indicates the $n-k$ value of H-DLC film before SR irradiation.

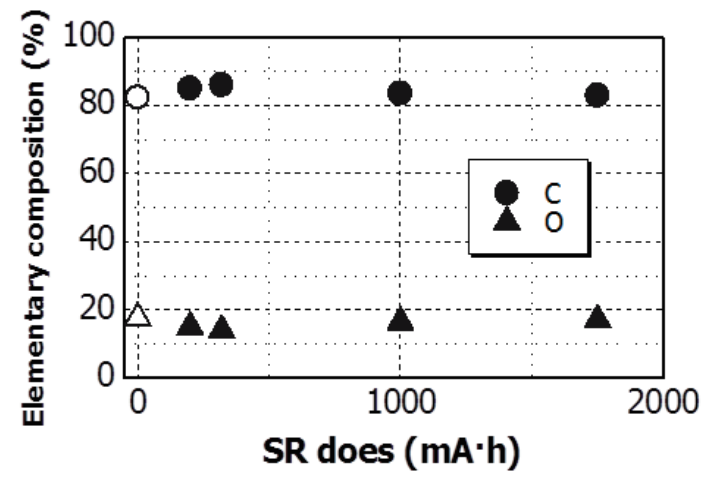

Fig. 7. Dependence of surface elementary composition of H-DLC film on SR dose. Circles and triangles indicate the fraction of carbon and oxygen, respectively. The open circle and triangle indicate the fraction of carbon and oxygen in the H-DLC film before SR irradiation, respectively.

thought to include not only the desorbed species from the sample but also residual gas species in the irradiation chamber and substrate Si wafer. Therefore, we measured the mass spectrum during SR exposure of the Si wafer without the deposition of DLC film as back-ground, and we subtracted these values from the measured mass spectrum during SR exposure of the H-DLC films. The mass spectrum just after the start of SR irradiation of a H-DLC film, which was obtained from the subtraction of background described above, is shown in Fig. 8. A peak was not observed for any mass greater than 60 .

The peaks in Fig. 8 at mass numbers 18 and 28 were ascribable to water molecules and nitrogen molecules, respectively. These are background species, which were not removed by subtraction, and/or species that adhered to the surface of the H-DLC films. The intense peak at mass number 2 showed that hydrogen molecules and/or hydrogen molecular ions were desorbed from the surface of the H-DLC film due to SR exposure. The peak at mass number 1 is due to hydrogen ions and/or hydrogen atoms, which may be directly emitted from the H-DLC film, and which possibly formed due to the decoupling of hydrogen molecules in the QMS. It should be noted that species with carbon bonded to hydrogen, such as $\mathrm{CH}^{+}$(mass number, 13), $\mathrm{CH}_{2}{ }^{+}$(mass number, 14), and $\mathrm{C}_{2} \mathrm{H}_{2}^{+}$(mass number 26) were not observed. In other words, desorption of carbon, namely etching, was much slower than desorption of hydrogen.

Next we investigated the dependence of the quantity of desorbed species on dose. In this experiment, we used commercial DLC films deposited by ion plating (Nanotec) as a representative of typical DLC films. The hydrogen content of ion-plating DLC (IP-DLC) film was $\approx 20 \%$, estimated using ERDA/RBS. Figure 9 shows the dose dependence of the most intense peak at mass number 2. A large quantity of hydrogen was emitted from the H-DLC film at the same time at which soft X-ray irradiation was started. The quantity of desorbed hydrogen decreased exponentially with increasing SR dose and became approximately constant at a very small value with an SR dose of more than $200 \mathrm{~mA} \cdot \mathrm{h}$. This SR dose dependence of the quantity of desorbed hydrogen from H-DLC film was consistent with that of the hydrogen content in the H-DLC films estimated using ERDA/RBS. ${ }^{(11)}$ On the other hand, desorption of hydrogen was hardly observed, 


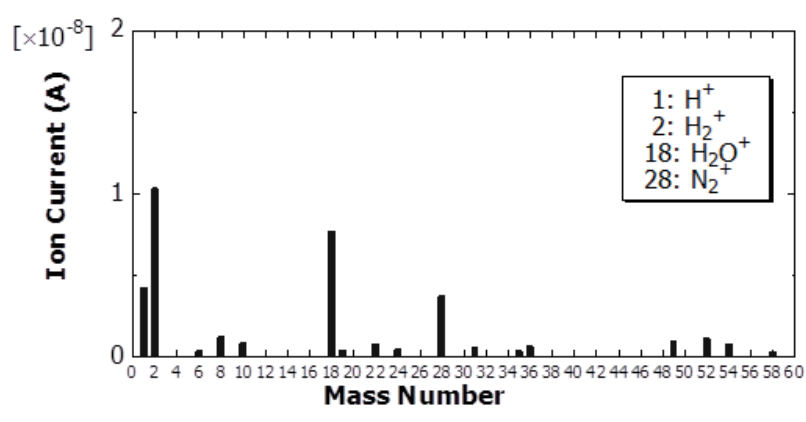

Fig. 8. Mass spectrum of desorbed species from H-DLC film just after irradiation started.

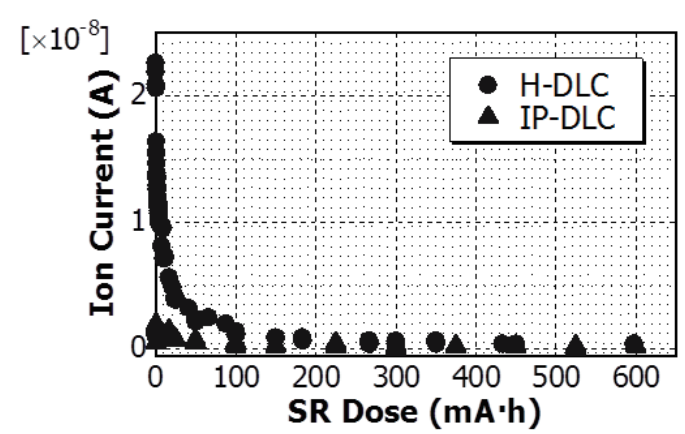

Fig. 9. Dependence of quantities of $\mathrm{H}_{2}{ }^{+}$from H-DLC (circles) and IP-DLC (triangles) films on SR dose.

even when IP-DLC film was exposed to soft X-rays. That is to say, we confirmed that hydrogen was not desorbed during SR exposure of DLC films with a hydrogen content less than a certain value. From these results, H-DLC film can be modified due to desorption of hydrogen by soft X-ray irradiation, while IP-DLC film has a high tolerance to soft X-rays.

\section{Discussion}

Various material and structural properties of H-DLC films were observed to change due to soft X-ray irradiation. The film thickness decreased with increasing SR dose, but it behaved in accordance with other properties, demonstrating a sudden change in the dose range of 0-200 mA.h; it remained almost constant at a dose of more than $200 \mathrm{~mA} \cdot \mathrm{h}$. These dose dependences agreed with those of the quantity of $\mathrm{H}_{2}{ }^{+}$emitted estimated using QMS, hardness, and hydrogen content in H-DLC films. ${ }^{(11)}$ Therefore, changes in these properties due to soft X-ray irradiation were undoubtedly related to hydrogen desorption from the H-DLC films. Film density, $s p^{2} /\left(s p^{2}+s p^{3}\right)$ ratio of carbon atoms, and optical constants $n$ and $k$ increased rapidly with an SR dose in the range of 0-1000 mA.h and remained almost constant with a dose larger than $1000 \mathrm{~mA} \cdot \mathrm{h}$. We considered that these changes were caused by the additional exposure of a H-DLC layer, lacking hydrogen to soft X-rays.

Carbon-hydrogen bonds in H-DLC films were decoupled by excitation due to SR exposure in the soft X-ray region. Decoupled hydrogen atoms were desorbed, while the remaining carbon atoms coupled with neighboring carbon atoms. As a result, the film volume decreased, and film density and hardness increased. In addition, the $s p^{2} /\left(s p^{2}+s p^{3}\right)$ ratio increased because some remaining carbon atoms formed carbon-carbon double bonds. It is worth noting that the surface elementary composition from XPS measurements did not show a change depending on SR dose, while the NEXAFS spectra depended strongly on SR dose. In addition, the desorbed species detected with the QMS did not include species containing carbon atoms. Therefore, the desorption rate of hydrogen was much faster than that of carbon during SR exposure of H-DLC films in the soft X-ray region. As a result, the decrease in film volume was confirmed by the increase in film density, not the etching of the film, which results in desorption of carbon from the film. No change 
in the surface elementary composition was ascribable to this process based only on desorption of hydrogen, which was not detected by XPS.

To estimate the penetration depth of soft X-rays into H-DLC films, transmittance was calculated using a calculation database. ${ }^{(36)}$ The density was supposed to be $1.25 \mathrm{~g} / \mathrm{cm}^{3}$, which was determined from XRR measurements. The transmittance at $300 \mathrm{eV}$, which corresponds to the energy of the carbon $K$ shell, was estimated to be $\approx 30 \%$ at a 200 -nm depth, which was the film thickness in this study. Therefore, the desorption of hydrogen occurred from the entire H-DLC film during our experiments.

\section{Conclusion}

We investigated the effect of soft X-ray radiation on highly hydrogenated DLC films. DLC film is known as a material that is tolerant to X-rays, but DLC film with high hydrogen content was found to be affected by soft X-ray irradiation. Various material and structural properties, e.g., density, hardness, optical constants, and $s p^{2} /\left(s p^{2}+s p^{3}\right)$ ratio, vary along with SR dose. These changes are caused by the desorption of hydrogen from the H-DLC film. Hydrogen atoms are desorbed by excitation due to soft X-ray irradiation, and the remaining carbon atoms bond to each other. As a result, the density and hardness increase and film volume decreases. From our estimation of soft X-ray transmittance in H-DLC film, desorption of hydrogen occurred not only near the surface but throughout the film. As a result, we observed large changes in optical constants. H-DLC film before SR exposure has characteristics of polymer-like carbon film, and after SR exposure its characteristics are those of a-C:H film.

\section{Acknowledgments}

Part of this work was supported by a Research Grant from Nippon Sheet Glass Foundation for Materials Science and Engineering. The authors thank the NewSUBARU staff for their efforts regarding the stable operation of the NewSUBARU ring.

\section{References}

1 S. Aisenberg and S. Chabor: J. Appl. Phys. 42 (1971) 3963.

2 J. Robertson: Surf. Coat. Technol. 50 (1992) 185.

3 A. Grill: Diamond Relat. Mater. 8 (1999) 428.

4 Y. Lifshitz: Diamond Relat. Mater. 8 (1999) 1659.

5 J. Robertson: Mat. Sci. Eng. B 37 (2002) 129.

6 C. Donnet, M. Belin, J. C. Augé, J. M. Martin, A. Grill, and V. Patel: Surf. Coat. Technol. 68-69 (1994) 626.

7 C. Donnet, J. Fontaine, T. Le Mogne, M. Belin, C. Héau, J. P. Terrat, F. Vaux, and G. Pont: Surf. Coat. Technol. 120-121 (1999) 548.

8 Tribology of Diamond-like Carbon, eds. C. Donnet and A. Erdemir (Springer, 2008).

9 H. Kyuragi and T. Urisu: Appl. Phys. Lett. 50 (1987) 1254.

10 K. Kanda, K. Yokota, M. Tagawa, M. Tode, Y. Teraoka, and S. Matsui: Jpn. J. Appl. Phys. 50 (2011) 055801.

11 R. Imai, A. Fujimoto, M. Okada, S. Matsui, T. Yokogawa, E. Miura, T. Yamasaki, T. Suzuki, and K. Kanda: Diamond Relat. Matter. 44 (2014) 8.

12 M. Hiratsuka, H. Nakamori, Y. Kogo, M. Sakurai, N. Ohtake, and H. Saitoh: J. Solid Mech. Eng. 7 (2013) 187.

13 T. Nakahigashi, Y. Tanaka, K. Miyake, and H. Oohara: Tribology Int. 37 (2004) 907.

14 A. Ando, S. Amano, S. Hashimoto, H. Kinoshita, S. Miyamoto, T. Mochizuki, M, Niibe, Y. Shoji, M. Terasawa, and T. Watanabe: Proc. IEE Particle Accelerator Conf. (1997) p. 757. 
15 K. Kanda, T. Ideta, Y. Haruyama, H. Ishigaki, and S. Matsui: Jpn. J. Appl. Phys. 42 (2003) 3983.

16 Y. Kato, K. Kanda, Y. Haruyama, and S. Matsui: Jpn. J. Appl. Phys. 43 (2004) 3938.

17 K. Edamatsu, Y. Tanaka, T. Yokokawa, K. Seki, M. Tohnan, T. Okada, and T. Ohta: Jpn. J. Appl. Phys. 30 (1991) 1073.

18 A. Gutiérrez, J. Díaz, and M.F. López: Appl. Phys. A 61 (1995) 111.

19 C. Lenardi, P. Piseri, V. Briois, C.E. Bottani, A. Li Bassi, and P. Milani: J. Appl. Phys. 85 (1999) 7159.

20 K. Kanda, T. Kitagawa, Y. Shimizugawa, Y. Haruyama, S. Matsui, M. Terasawa, H. Tsubakino, I. Yamada, T. Gejo, and M. Kamada: Jpn. J. Appl. Phys. 41 (2002) 4295.

21 A. Saikubo, K. Kanda, M. Niibe, and S. Matsui: New Diamond Front. Carbon Technol. 16 (2006) 235.

22 A. Saikubo, N. Yamada, K. Kanda, S. Matsui, T. Suzuki, K. Niihara, and H. Saitoh: Diamond Relat. Mater. 17 (2008) 1743.

23 M. Niibe, M. Mukai, H. Kimura, and Y. Shoji: AIP Conf. Proc. 705 (2004) 243.

24 M. Niibe, M. Mukai, S. Miyamoto, Y. Shoji, S. Hashimoto, A. Ando, T. Tanaka, M. Miyai, and H. Kitamura: AIP Conf. Proc. 705 (2004) 576.

25 P. E. Batson: Phys. Rev. B 48 (1993) 2608.

26 N. Savvides: J. Appl. Phys. 59 (1986) 4133.

27 F. Demichelis, C.F. Pirri, and A. Tagliaferro: Phys. Rev. B 45 (1992) 323.

28 B.K. Tay, X. Shi, L.K. Cheah, and D.I. Flynn: Thin Solid Films 308-309 (1997) 268.

29 J. Lee, R. W. Collins, V. S. Veerasamy, and J. Robertson: J. Non-Cryst. Solids 227-230 (1998) 617.

30 W. S. Guo, S. P. Wong, and Y. H. Yu: Nucl. Inst. Meth. B, 169 (2000) 54.

31 A. Canillas, M. C. Polo, J. L. Andújar, J. Sancho, S. Bosch, J. Robertson, and W. I. Milne: Dia. Relat. Mat. 10 (2001) 1132.

32 W. J. Li, Z. R. Song, Y. H. Yu, X. Wang, S. C. Zou, and D. S. Shen: J. Appl. Phys. 94 (2003) 284.

33 M. Vinnichenko, R. Gago, N. Huang, Y. X. Leng, H. Sun, U. Kreissig, M. P. Kulish, and M. F. Maitz: Thin Solid Films 455-456 (2004) 530.

34 I. Ohlídal, D. Nečas, D. Franta, and V. Buršíková: Dia. Relat. Mat. 18 (2009) 364.

35 M. Hiratsuka, H. Nakamori, Y. Kogo, M. Sakurai, N. Ohtake, and H. Saitoh: J. Solid. Mechanics and Materials Engineering, Special Issue on the Asian Symp. on Materials \& Processing 2012, 7, pp. 187-198 (2013).

36 The Center for X-Ray Optics at Lawrence Berkeley National Laboratory: X-Ray Database, http://henke.lbl. gov/optical_constants/. 